\title{
Evolution and diversity of community-associated methicillin-resistant Staphylococcus aureus in a geographical region
}

Geoffrey W Coombs ${ }^{1,2^{*}}$, Stefan Monecke ${ }^{3}$, Julie C Pearson ${ }^{1}$, Hui-leen Tan ${ }^{1}$, Yi-Kong Chew ${ }^{1}$, Lynne Wilson ${ }^{1}$, Ralf Ehricht ${ }^{3}$, Frances G O'Brien ${ }^{2}$ and Keryn J Christiansen ${ }^{1,2}$

\begin{abstract}
Background: Community-associated methicillin-resistant Staphylococcus aureus (CA-MRSA) was first reported in remote regions of Western Australia and is now the predominant MRSA isolated in the state. The objective of this study is to determine the genetic relatedness of Western Australian CA-MRSA clones within different multilocus sequence type (MLST) clonal clusters providing an insight into the frequency of S. aureus SCCmec acquisition within a region.

Results: The CA-MRSA population in Western Australia is genetically diverse consisting of 83 unique pulsed-field gel electrophoresis strains from which 46 MLSTs have been characterised. Forty five of these sequence types are from 18 MLST clonal clusters and two singletons. While SCCmec IV and V are the predominant SCCmec elements, SCCmec VIII and several novel and composite SCCmec elements are present. The emergence of MRSA in diverse $S$. aureus clonal clusters suggests horizontal transmission of the SCCmec element has occurred on multiple occasions. Furthermore DNA microarray and spa typing suggests horizontal transfer of SCCmec elements has also occurred within the same CC. For many single and double locus variant CA-MRSA clones only a few isolates have been detected.
\end{abstract}

Conclusions: Although multiple CA-MRSA clones have evolved in the Western Australian community only three clones have successfully adapted to the Western Australian community environment. These data suggest the successful evolution of a CA-MRSA clone may not only depend on the mobility of the SCCmec element but also on other genetic determinants.

\section{Background}

Based on phenotypic and genotypic typing methods, community onset methicillin-resistant Staphylococcus aureus infections are caused by healthcare-associated MRSA (HA-MRSA) strains, which appear to have been transferred from hospitals or healthcare facilities into the community by patients or healthcare workers [1], or by community-associated MRSA (CA-MRSA) strains, which have been isolated from people who have had little or no contact with healthcare facilities or healthcare

\footnotetext{
* Correspondence: geoffrey.coombs@health.wa.gov.au

'Australian Collaborating Centre for Enterococcus and Staphylococcus Species (ACCESS) Typing and Research. PathWest Laboratory Medicine - WA, Royal Perth Hospital, Wellington Street, Perth, Western Australia, 6000 Australia

Full list of author information is available at the end of the article
}

workers [2]. This distinction between community and healthcare facility however has become blurred with the replacement of HA-MRSA with CA-MRSA in hospitals $[3,4]$.

In contrast to HA-MRSA, CA-MRSA strains are generally more susceptible to non beta-lactam antibiotics, grow significantly faster, have different clonal backgrounds, carry smaller staphylococcal cassette chromosome mec (SCCmec) elements (most commonly SCCmec type IV or type V), have enhanced virulence properties and frequently harbor genes expressing Panton-Valentine leukocidin (PVL) [5-8]. Rather than a worldwide spread of a single clone multiple CA-MRSA clones have emerged from diverse genetic backgrounds. Several well characterized CA-MRSA clones predominate in different regions: Sequence type (ST) 8-IV [2B]
C Biomed Central

() 2011 Coombs et al; licensee BioMed Central Ltd. This is an Open Access article distributed under the terms of the Creative Commons Attribution License (http://creativecommons.org/licenses/by/2.0), which permits unrestricted use, distribution, and reproduction in any medium, provided the original work is properly cited. 
(USA300) and ST1-IV [2B] (USA400) in North America [9,10]; ST80-IV [2B] (European clone) in Europe [8], North Africa [11] and the Middle East [12]; ST59-V [5C2\&5] (Taiwan clone) in Taiwan [13]; ST93-IV [2B] (Queensland clone) in Australia [14], ST30-IV [2B] (South West Pacific [SWP] CA-MRSA) in the Western Pacific [15,16], and ST772-V [5C2] (Bengal Bay clone) in India and Bangladesh [17]. Transmission of these clones into other regions has occurred [18,19]. This occurrence of concurrent epidemics of CA-MRSA in many countries by different clones has been striking. Equally noteworthy are a number of common features of these epidemics, prominent among them the ability to cause severe infections in young otherwise healthy people and the carriage of the $l u k F-P V / l u k S-P V$ PVL encoding genes by the organism.

The earliest report of CA-MRSA infections involved indigenous people living in remote communities in the sparsely populated Kimberley region of Western Australia (WA) [20]. Approximately 50\% of the people in this region are indigenous, many of whom live in poor socioeconomic conditions. Infected skin lesions and staphylococcal sepsis occur frequently and empirical antistaphylococcal therapy is often prescribed. Colloquially known as "WA-MRSA", the early isolates have a similar pulsed-field gel electrophoresis (PFGE) pattern and have subsequently been characterized as a single clone; PVL-negative WA5 (ST8-IV/spa t008) [21]. By 200622 CA-MRSA clones were identified in WA, with PVL-negative WA 1 (ST1-IV [2B]/t127) replacing WA5 as the predominant clone [22]. At this time CA-MRSA from indigenous people living in remote areas outside of WA were reported in the Northern Territory [23], Queensland [24] and Central Australia [25]. As may be expected in a geographically large country with relatively few dense concentrations of population, often separated by large areas of desert, different CA-MRSA clones evolved in these communities.

In 1982 colonization or infection with MRSA became a notifiable condition in WA. For infection control purposes all MRSA isolated in the state since 1997 have been referred to the Australian Collaborating Centre for Enterococcus and Staphylococcus Species (ACCESS) Typing and Research where based on molecular markers they are characterized as either HA-MRSA or CAMRSA [26]. Although a state-wide policy of screening all patients and healthcare workers who have lived outside the state for MRSA has prevented HA-MRSA from becoming endemic in Western Australian hospitals, it has not prevented CA-MRSA from becoming established in the community. In WA the public health system is divided into two metropolitan health regions and seven country health regions. The state encompasses an area of 1.02 million square miles and has a population of approximately 2.24 million people. In 1983 , the overall rate of MRSA notifications was 10 per 100,000 persons in the rural country health regions and 7/100,000 in the metropolitan regions [27]. By 2006 notifications rates throughout the state had increased to $179 / 100,000$ persons of which $144 / 100,000$ were CA-MRSA. In the metropolitan health regions the CA-MRSA notification rate was 134/100,000 whilst in the Kimberley health region the CA-MRSA notification rate had increased 40fold to $391 / 100,000$ [18].

CA-MRSA is thought to emerge when a locally prevalent strain of methicillin susceptible $S$. aureus (MSSA) acquires a SCCmec element and utilizes mobile genetic elements and single nucleotide polymorphisms to establish local and geographic niches [28]. As WA is a remote region in which all MRSA isolates are referred to a central typing laboratory it is an ideal environment to study the emergence and evolution of CA-MRSA. MLST, SCCmec, spa typing and microarray DNA is performed on all isolates with a unique PFGE pulsotype. The aim of this study is to determine the genetic relatedness of WA CA-MRSA clones within different MLST clonal clusters (CC) providing an insight into the frequency of $S$. aureus SCCmec acquisition within a region. The genetic profile of these clones may also offer an explanation why only a few WA CA-MRSA clones have successfully adapted to the community environment.

\section{Results}

The 83 unique PFGE strains isolated in Western Australia from 1989 to 2010 were $n u c$ and $m e c A$ gene positive by PCR. The DNA microarray $S$. aureus species markers gapA (glyceraldehyde 3-phosphate dehydrogenase) and rrn STAU (S. aureus ribosomal marker) were detected in all strains. The array's linear primer elongation method detected the katA (catalase A), coA (coagulase), $n u c$, spa (protein A) and sbi (IgG-binding protein) $S$. aureus species markers in 78 strains. These markers were either not detected or detected only by random amplification in five strains (WA8, WA47, WA72, WA76 and WA79).

Forty six STs were identified by MLST. Using the MLST website's eBURST V3 algorithm 45 STs were grouped into $18 \mathrm{CCs}$ and two singletons (Figure 1). The CC for WA76 (ST1303) has not been determined.

Several SCCmec types and subtypes, novel SCCmecs, and composite SCCmecs were identified. Forty five strains harbor SCCmec IVa-d [2B] (31 IVa, 2 IVb, 9 IVc, 3 IVd), 12 strains SCCmec $\mathrm{V}$ [5C2] and two strains SCCmec VIII [4A]. Two strains have non typeable SCCmec IV subtypes and four strains have a SCCmec element with a novel ccr gene complex including three with a class B mec gene complex and one with a class A mec complex. Eighteen strains 


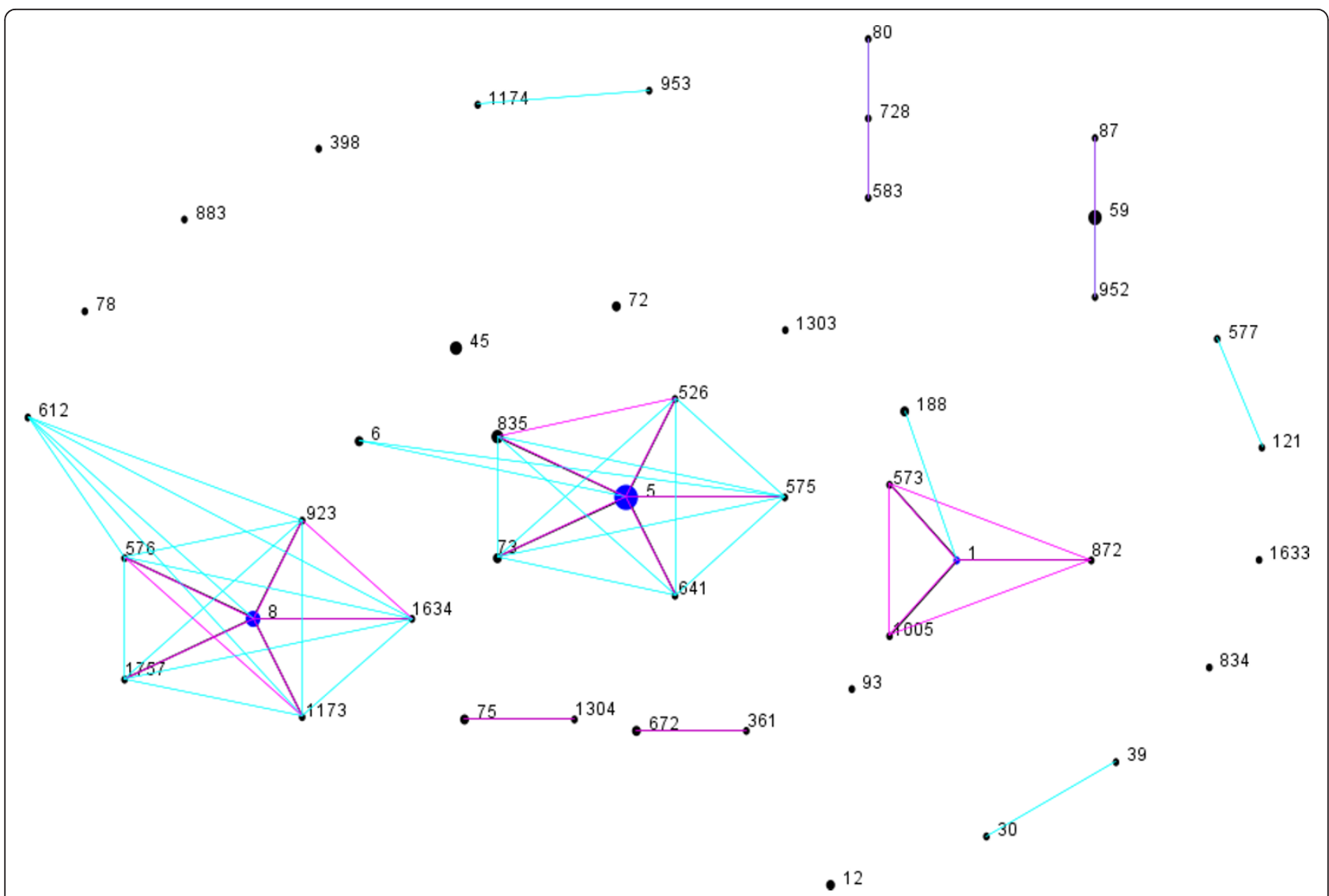

Figure 1 eBURST generated population snapshot of CA-MRSA clones isolated in Western Australia (http://www.mlst.net/). Each sequence types (STs) is represented by a black dot. The ancestral ST of a clonal complex is represented by a blue dot. The size of the dot reflects the number of WA CA-MRSA clones with this ST. STs that diverge at no more than one of the seven MLST loci belong to the same clonal complex. Double locus variants (DLVs) are included if the linking single locus variant (SLV) was present in the MLST database. SLVs and DLVs of a sequence type are represented by pink and blue line respectively. Purple lines represent overlapping pink and blue lines.

harbor SCCmec elements with composite ccr gene complexes including 12 with SCCmec V [5C2\&5] (5C2 plus $c c r C 1$ allele 8), three with SCCmec IVa [2B]\&5 (2B plus a type $5 \mathrm{ccr}$ gene complex), one with $\mathrm{V}(5 \mathrm{C} 2)$ $\& 2(5 \mathrm{C} 2$ plus a type $2 \mathrm{ccr}$ gene complex) and two with $\mathrm{V}$ [5C2\&5]\&2 (a composite SCCmec V element plus a type 2 ccr gene complex).

The MLST, spa type, agr type, capsule type, SCCmec, antibiogram, resistance genotype, lukF/S-PVL genes, enterotoxin genes and bacteriophage associated virulence genes of each unique PFGE strain are provided in Additional File 1. Information on target genes, probes, and primers is provided in Additional File 2. Complete hybridization profiles for the individual strains can be provided on request.

\section{Clonal Complex 1}

CC1 contains five strains including the PVL positive Bengal Bay clone (ST772 [a single locus variant \{slv\} of ST1]-V [5C2]/t3387). This strain is epidemiologically linked to a healthcare worker from India and is not considered a WA CA-MRSA.

Based on the agr/capsule and SCCmec type, the remaining four strains are divided into two groups:

\section{Group 1}

agr type III/capsule type 8 SCCmec IVa [2B] contains PVL negative WA1 (ST1/t127), WA45 (ST872 [slv of ST1]/t127), and WA57 (ST1005 [ST1 slv]/t127). WA1 and WA45 harbor a $c c r A-1$ and $c c B-1$ gene complex and Q6GD50 (fusidic acid resistance marker) indicating the presence of the mobile fusidic acid SCC element SCCfur. WA1 is known to carry multiple plasmids such as a 2-kb plasmid encoding resistance to erythromycin [29] and this presumably accounts for the differences in the antibiogram and resistance genotype for WA1, WA45 and WA57. In addition to enterotoxin genes the three strains harbor a type D immune evasion cluster [IEC] $(s e A+s a k+s c n)$ [30]. Group 2

agr type II/capsule type $5 \mathrm{SCCmec} \mathrm{V}$ [5C2] contains PVL negative WA10 (ST573 [ST1 slv]/t5073. WA10 
carries several enterotoxin genes including the enterotoxin egc cluster $[s e G+s e I+s e M+s e N+s e O+s e U / Y])$. Unlike WA1, WA45 and WA57, WA10 does not carry the type D IEC, the pathogenicity island harboring the leukocidin D/E component, the protease splA gene and the $h s d S$ gene. The $s s l / s e t$ genes and cell surface adhesions encoding genes of WA10 are closely related to the Bengal Bay clone.

\section{Clonal Complex 5}

CC5 contains 27 strains. Based on the agr/capsule type the isolates are divided into two groups which are further divided into subgroups based on the SCCmec type.

\section{Group 1}

agr type $\mathrm{I} /$ capsule type 8 (2 strains)

i. SCCmec IVa [2B] contains WA51 (ST6 [ST5 dlv]). The protein A variable region in WA51 could not be amplified and therefore a spa type cannot be allocated.

ii. SCCmec IVa [2B]\&5 contains WA66 (ST6/ $\mathrm{t701).}$

WA51 and WA66 harbor a type D IEC Neither strain harbors the $l u k F-P V / l u k S-P V$ PVL encoding genes.

\section{Group 2}

agr type II/capsule type 5 (25 strains)

Unlike Group 1 strains, these 25 strains harbour the enterotoxin egc cluster. Ten spa types were identified, of which nine are closely related: t002, t045, t071, t442, t688, t1265, t2666, t3378, t4065.

i. SCCmec IVa [2B] contains WA3 (ST5/t002), WA64 (ST5/t3778), WA71 (ST5/t002), WA82 (ST5/t002), WA25 (ST575 [ST5slv]/t002), WA50 (ST73 [ST5slv]/t002) and WA65 (ST73/t002). PVL negative WA3, WA71, WA82, WA25, WA50 and WA65 harbor a type F IEC $(s e P+s a k+c h p+s c n)$. PVL positive WA64 harbors a type A IEC (seA+sak $+c h p+s c n)$. WA64 and WA65 also harbor edinA (epidermal cell differentiation inhibitor A gene).

ii. SCCmec IVc [2B] contains PVL negative WA74 (ST5/t002) which harbors a type F IEC.

iii. SCCmec IV [2B] contains PVL negative WA39 (ST526 [ST5slv]/t4065) which has a non typeable SCCmec IV [2B] element and a type B IEC $(s a k+c h p+s c n)$.

iv. SCCmec V [5C2] contains PVL negative WA14 (ST5/t442), WA35 (ST5/t688), WA81 (ST5/t045) [a non related spa type] and WA90 (ST5/t1265). WA81 harbors a type F IEC; WA14 and WA90 a type G IEC $(s e P+s e k+s c n)$ and WA35 a type B IEC. v. SCCmec V [5C2\&5] contains PVL negative WA11 (ST5/t045), WA86 (ST5/t002), WA34 (ST5/t458), WA80 (ST5/t071), WA85 (ST5/ t2666), and WA87 (ST835 [ST5slv]/t002). WA85 and WA86 harbor a type F IEC; WA34, WA80 and WA87 a type B IEC and WA11 a type E IEC $(s a k+s c n)$. WA80 harbors the ACME (arginine catabolic mobile element) genes.

vi. SCCmec V [5C2]\&2 contains PVL negative WA61 (ST641 [ST5slv]/t002) which harbors a type E IEC.

vii. SCCmec V [5C2\&5]\&2 contains PVL negative WA40 (ST835 [ST5slv]/t002) and WA46 (ST835/ t002). WA40 harbors a type B IEC while WA46 a type E IEC.

viii. SCCmec novel [novel B] contains PVL negative WA18 (ST5/t002), WA21 (ST5/t002) and WA48 (ST835/t002) harboring $\operatorname{ccr} A-1$ and a class B mec complex (mecA and a truncated mecR1 genes). WA18 harbors a type F IEC; WA21 a type D IEC; and WA48 a type B IEC.

\section{Clonal Complex 8}

The 12 CC8 strains are all agr type I/capsule type 5. Seven closely related spa types were identified: t008, t024, t064, t334, t711, t1635, t2238.

The CC8 strains include the ST8-MRSA-IVc [2B]/t008 USA300 MRSA clone [31]. Based on the SCCmec type the remaining 11 strains are divided into seven subgroups:

i. SCCmec IVa [2B] contains WA5 (ST8/t008), WA6 (ST8/t008), WA62 (ST923 [ST8slv]/t1635), and WA83 (ST1634 [ST8slv]/t711). WA5, WA62, and WA83 harbor a type B IEC. An IEC was not detected in WA6. Unlike the other WA CC8 strains, WA62 is PVL positive.

ii. SCCmec IVd [2B] contains WA58 (ST1173 [ST8slv]/t064) and WA20 (ST612 [ST8dlv]/t064) which harbor a type D IEC.

iii. SCCmec IVa [2B]\&5 contains WA92 (ST1757 [ST8slv]/t024) which does not harbor an IEC.

iv. SCCmec IV [2B] contains WA31 (ST576 [ST8slv]/t334) which does not harbor an IEC. The SCCmec IV element is non typeable.

v. SCCmec V [5C2] contains WA77 (ST8/t008) which harbors a type D IEC, the ACME determinant, and SCCfus.

vi. SCCmec V ([5C2\&5]) contains WA53 (ST8/ t2238) which harbors a type D IEC.

vii. SCCmec VIII (4A) contains WA16 (ST8/t024) which harbors a type D IEC. 


\section{Clonal Complex 12}

CC12 contains two agr group II/capsule type 8 strains which harbor a type G IEC. Neither strain harbor the lukF-PV/lukS-PV PVL encoding genes.

Based on the SCCmec type the two strains are divided into two subgroups:

i. SCCmec IVa [2B] contains WA69 (ST12/t160).

ii. SCCmec novelA contains WA59 (ST12/t160) which harbors a class A mec complex (mecA, complete mecR1 and mecI regulatory genes). The $c c r$ genes were not detected by DNA microarray and did not amplify with PCR primers.

\section{Clonal Complex 30}

CC30 contains two agr group III/capsule type 8 strains: PVL positive ST30-IVc [2B]/t019 and PVL negative WA68 (ST39 [ST30dlv]-IVc [2B]/t2643). Their protease, haemolysin, leukocidin, ssl/set, hsdS, and cell surface adhesion profiles are not homogeneous and their spa types are not closely related.

The DNA microarray profile of ST30-IVc [2B]/t019 is homogeneous with the South Western Pacific (SWP) ST30-IV clone as is therefore not considered a WA CAMRSA.

WA68 harbors a type D IEC and tst-1genes.

\section{Clonal Complex 45}

CC45 contains four PVL negative strains. Based on the agr group/capsule type the four isolates are divided into two groups which are further divided into subgroups based on the SCCmec type.

\section{Group 1}

agr group I/capsule 8 (two strains)

i. SCCmec IVa [2B] contains WA75 (ST45/t1424).

ii. SCCmec V [5C2] contains WA4 (ST45/t123)

which harbors tst1 genes.

Both strains harbor a type B IEC. The spa types are not closely related.

\section{Group 2}

agr group IV/capsule type 8 (two strains)

i. SCCmec IVc [2B] contains WA23 (ST45/t1575)

ii. SCCmec V [5C2\&5] contains WA84 (ST45/t1081). Both strains harbor a type B IEC and closely related spa types.

\section{Clonal Complex 59}

CC59 agr type I/capsule type 8 contains seven strains.

The DNA microarray profiles of ST59/ST $952-\mathrm{V}$ [5C2\&5] t437/t1950 are homogeneous with the Taiwan clone and therefore are not considered WA CA-MRSA [32].

Based on the SCCmec types the remaining five strains are divided into three subgroups:

i. SCCmec IVa [2B] contains PVL positive WA55 and WA56 (ST59/t437). WA55 harbors a type B IEC while WA56 a type A IEC.

ii. SCCmec IVb [2B] contains two PVL negative strains with unrelated spa types: WA73 (ST59/t528) and WA24 (ST87 [ST59slv]/t216). WA73 harbors a type C IEC $(c h p+s c n)$ and WA24 a type B IEC.

iii. SCCmec IVa $[2 \mathrm{~B}] \& 5$ contains PVL negative WA15 (ST59/t976) which harbors a type A IEC.

\section{Clonal Complex 72}

CC72 contains two agr group I/capsule type 5 strains with closely related spa types. Based on the SCCmec type the two strains are divided into two subgroups:

i. SCCmec IVa [2B] contains PVL positive WA44 (ST72/t791) harboring a type B IEC.

ii. SCCmec V (5C2) contains PVL negative WA91

(ST72/t3092) harboring a type E IEC and tst1 genes.

\section{Clonal Complex 75}

CC75 contains three PVL negative strains which are agr group/capsule nontypeable by DNA microarray: WA8 (ST75-IVa [2B]), WA79 (ST75-IVa [2B]) and WA72 (ST1304 [ST75slv]-IVa [2B]) [33]. The three strains have the same spa sequence (259-23-23-17-17-17-23-23-2317-16) which has not been allocated a spa type number by the Ridom website. The three strains harbor a type $\mathrm{E}$ IEC.

\section{Clonal Complex 80}

CC80 contains three PVL positive agr group III/capsule type 8 strains: ST80-IVc [2B]/t044, ST583 [ST80slv]-IVc [2B]/t044, and ST728 [ST80slv]-IVc [2B]/t044. The DNA microarray virulence profiles are identical with the European ST80-IV [2B] clone and therefore the three strains are not considered WA CA-MRSA.

\section{Clonal Complex 97}

CC97 contains two PVL negative agr group I/capsule type 5 strains with closely related spa types: WA54 (ST953 [ST97dlv]-IVa [2B]/t359) and WA63 (ST1174[ST97dlv]IVa [2B]/t267). The strains harbor a type E IEC.

\section{Clonal Complex 121}

CC121 contains two PVL negative agr group IV/capsule type 8 strains with closely related spa types. The two 
strains harbor a type E IEC and based on the SCCmec type, are divided into two subgroups:

i. SCCmec V [5C2] contains WA22 (ST577 [ST121 $\mathrm{dlv}] / \mathrm{t} 3025$ ) which harbors etA (exfoliative toxin serotype A) and edinA genes.

ii. SCCmec V [5C2\&5] contains WA93 (ST121/t159).

\section{Clonal Complex 188}

CC188 contains two PVL negative agr group I/capsule type 8 strains: WA38 and WA78 (ST188-IVa [2B]/t189). The two strains have a type B IEC.

\section{Clonal Complex 361}

CC361 contains three PVL negative agr group I/capsule type 8 strains. The spa types are closely related. Based on the SCCmec type the three strains are divided into three subgroups:

i. SCCmec IVa [2B] contains WA29 (ST672 (ST361slv)/t1309) which harbors a type E IEC and tst1 genes.

ii. SCCmec V [5C2] contains WA70 (ST672/t1309).

iii. SCCmec VIII [4A] contains WA28 (ST361/t315)

which harbors a type B IEC.

The following CCs contained a single strain:

\section{Clonal Complex 9}

PVL negative WA13 (ST834-IVc [2B]/t3029) is agr group I/capsule type 8 and harbors a type B IEC and tst1 genes.

\section{Clonal Complex 88}

PVL negative WA2 (ST78-IVa [2B]/t3205) is agr group III/capsule type 8 and harbors a type B IEC.

\section{Clonal Complex 152}

PVL positive WA89 (ST1633-V [5C2]/t355) is agr group I/ capsule type 5 and harbors a type E IEC and edinB genes.

\section{Clonal Complex 398}

Although PVL negative ST398-V [5C2\&5]/t034 is frequently associated with livestock, the strain is increasingly isolated from human patients [34]. Rarely identified in Australia, the DNA microarray profile of this isolate is homogeneous with the European livestock-associated ST398 strain and is therefore not considered a WA CA-MRSA.

\section{WA76 (Clonal Complex not Determined)}

PVL negative WA76 (ST1303-IVa [2B]) is agr group III with a non typeable capsule by DNA microarray. The spa sequence (259-25-17-17-16-16-16-16) has not been allocated a spa type number by the Ridom website.

\section{Queensland Clone (Singleton)}

PVL positive ST93-IVa [2B]/t202 is agr group III/capsule type 8 and harbors a type B IEC. The DNA microarray profile is homogeneous with the Queensland clone. Due to its origin and widespread distribution outside WA the Queensland clone is not considered a WA CA-MRSA.

\section{WA47 (Singleton)}

PVL negative WA47 (ST883-IVd [2B]/t7462) has a non typeable agr group/capsule type by DNA microarray.

\section{Discussion}

As all MRSA isolated in WA are referred to a central typing laboratory it is possible to investigate the emergence and evolution of CA-MRSA in a remote region.

Prior to the global evolution and expansion of CAMRSA, five CA-MRSA clones were identified in the indigenous population living in the remote communities of the sparsely populated Kimberley, Pilbara and Eastern Goldfield regions of WA [29]. These five PVL negative clones include: WA1 (CC1: ST1-IVa [2B]/t127), WA2 (CC88: ST78-IVa [2B]/t3205), WA3 (CC5: ST5-IVa [2B]/t002), WA4 (CC45 ST45-V (5C2)/t123) and WA5 (CC8: ST8-IVa [2B]/t008). WA5 and WA1 were originally isolated from clinical specimens in 1989 and 1995 respectively, and WA2, WA3 and WA4 from nasal carriage specimens in 1995. The emergence of CA-MRSA clones in different MLST clonal clusters indicates horizontal transmission of the SCCmec element into S. aureus has occurred on at least five occasions in these remote communities: SCCmec IVa [2B] into CC1 (ST1), CC5 (ST5), CC8 (ST8), CC88 (ST78), and SCCmec V [5C2] into CC45 (ST45). Based upon the spa type and the DNA microarray profile at least six evolutionary events have occurred on at least three occasions from these clones (ie vertical transmission of the SCCmec element): twice from WA1, WA3 and WA5 (Figure 2). Vertical transmission of the SCCmec element has not been identified for WA4 or WA2.

The emergence of WA1, WA2 and WA3 has been due to the acquisition and insertion of the small and highly mobile type IVa [2B] SCCmec element, presumably harbored by methicillin resistant coagulase negative staphylococci (MRCNS). Several hypotheses to explain the transmission of a SCCmec element from MRCNS to $S$. aureus have been proposed including the increased use of antimicrobials within a community [35]. Many of the Kimberley indigenous population live in poor socioeconomic conditions. Staphylococcal skin lesions, commonly resulting from scabies infestation, trachoma and venereal 


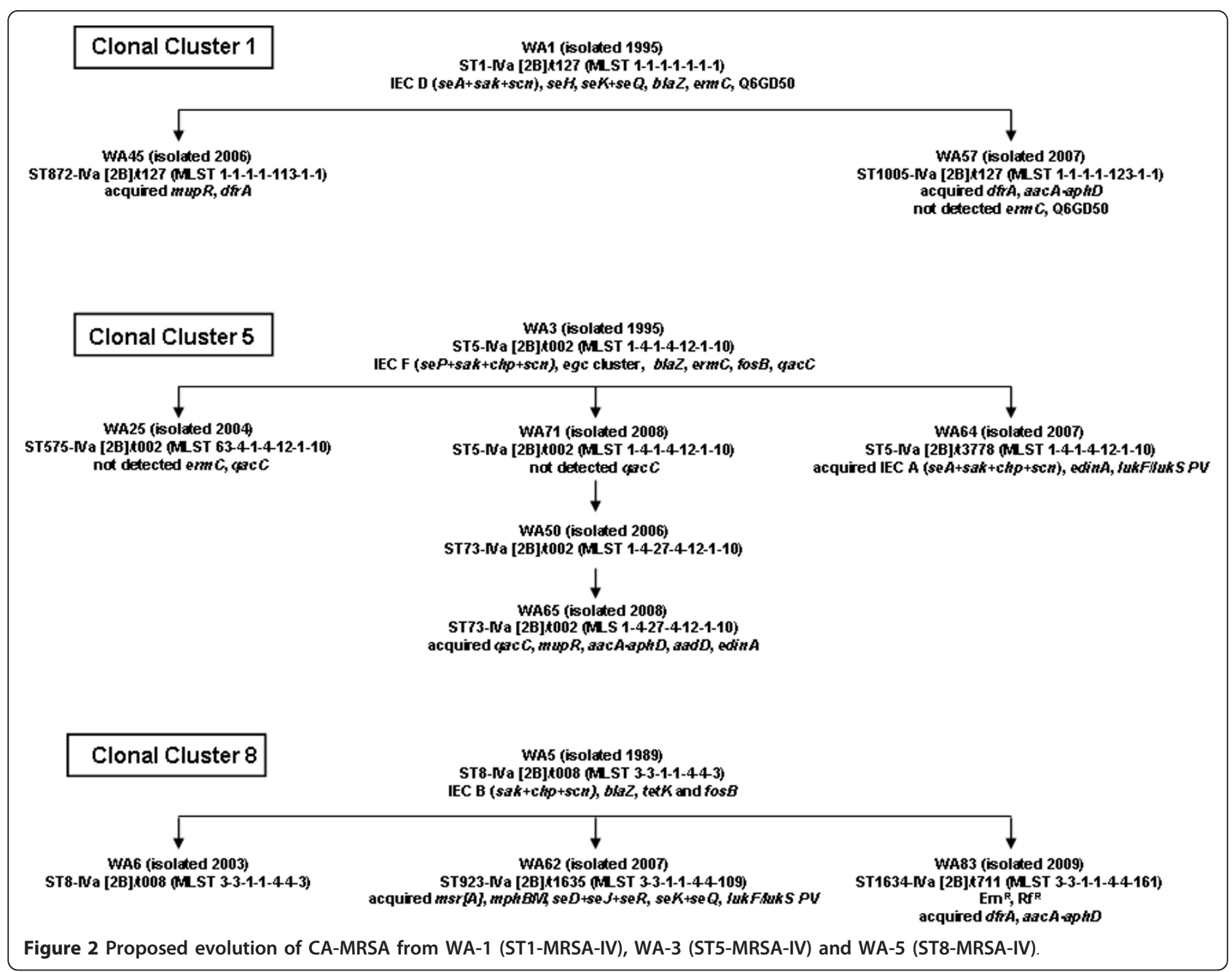

diseases such as chlamydia and gonorrhea occur frequently in this population. Consequently empirical therapy using $\beta$-lactamase stable penicillins and azithromycin is often prescribed [36]. The frequent use of these antimicrobials may have assisted in the acquisition of the SCCmec element and erm genes into S. aureus. Genetic studies however have shown these newly emerged CA-MRSA clones did not originate in the predominant methicillinsusceptible S. aureus (MSSA) clones found in these communities, suggesting not all clones are able to acquire or retain the SCCmec element [37]. The subsequent dissemination of WA1, WA2 and WA3 into the wider community suggests the acquisition of the SCCmec element and the erm genes has given these clones a selective advantage. WA4 and WA5 however have not been successful in spreading beyond the indigenous communities suggesting the acquisition of the SCCmec element does not provide a universal selective advantage.

Many of the remaining 46 CA-MRSA clones, identified between July 2003 and June 2010, were not isolated in remote WA indigenous communities. The geographical spread of CA-MRSA over long distances and across cultural borders is believed to be a rare event compared to the frequency in which the SCCmec element is acquired by $S$. aureus [38]. Most of these clones are therefore likely to have evolved in WA. Some clones are slvs and dlvs of pre-existing CA-MRSA, and their SCCmec type, spa type and DNA microarray profile suggests vertical transmission of the SCCmec element has occurred. However the emergence of MRSA in several unrelated $S$. aureus clonal clusters suggests horizontal transmission of the SCCmec element has also occurred. SCCmec typing and spa typing and DNA microarray results also suggests horizontal transfer of SCCmec elements has occurred into the same $\mathrm{CC}$ on more than one occasion.

Although several SCCmec elements have been acquired by multiple $S$. aureus clones from which many CA-MRSA clones have emerged, only a few clones have successfully adapted to the WA community 
environment. Between July 2009 to June 2010 4,691 MRSA were referred to ACCESS Typing and Research of which 3,931 were characterized as CA-MRSA. Overall $84 \%(3,024)$ of isolates were from clinical infections and the 16\% (907) from colonized patients. Approximately $88 \%$ of CA-MRSA were identified as WA1 (40\%), WA2 (24\%) and WA3 (8\%). For most clones, including WA4 and WA5 only a few isolates were detected. (http:// www.public.health.wa.gov.au/3/896/3/camrsa.pm).

For many slv and dlv CA-MRSA only a small number of isolates have been detected suggesting changes in the housekeeping genes may have conferred a fitness cost or did not allow the SCCmec element to be maintained. For example WA45 and WA57 are slvs of ST1 and their SCCmec and spa type and DNA microarray profile suggest they have evolved from WA1 (Figure 2). WA45 was first identified in 2006 and WA57 in 2007. Although WA1 has become the most successful CA-MRSA clone in the WA community only one isolate of WA45 and two isolates of WA56 have so far been identified (http:// www.public.health.wa.gov.au/3/896/3/camrsa.pm).

Six PVL positive pandemic CA-MRSA clones (plus three closely related clones) have been isolated in WA: Bengal Bay CA-MRSA (ST772-V [5C2]/t3387), USA300 MRSA (ST8-IVc [2B]/t008), SWP CA-MRSA (ST30-IVc [2B]/t019), Taiwan CA-MRSA (ST59-V [5C2\&5]/t437 and the slv ST952-V [5C2\&5]/t1950), European CAMRSA (ST80-IVc [2B]/t044 and the slvs, ST583-IVc [2B]/t044 and ST728-IVc [2B]/t044), and the Queensland CA-MRSA (ST93-IVa [2B]/t202). The epidemiology of the USA300 and Taiwan CA-MRSA clones in WA and the Queensland and SWP CA-MRSA clones in Australia have previously been reported $[18,31,32]$. Patients colonized or infected with the Bengal Bay clone have been observed to be epidemiologically linked to Indian healthcare workers (unpublished data). The USA300, European, Taiwanese and Bengal Bay CA-MRSA clones are not frequently isolated in WA. This may be due, in part, to WA Health Department infection control interventions applied to patients who are colonized or infected with international PVL positive pandemic clones. A seventh pandemic clone has recently been identified. The DNA microarray profile and the SCCmec element of the PVL negative ST398-V [5C2\&5] is indistinguishable from the pandemic ST398 clone initially isolated from pigs and pig farmers in the Netherlands [39]. Only one isolate, from a patient with travel outside of Australia, has been identified in WA.

The Queensland clone (ST93-IVa [2B]) first detected on the east coast of Australia in the Caucasian population in 2000 [40], has become one of the most prevalent CA-MRSA isolated in Australia [18] and in 2010 accounted for $18 \%$ of CA-MRSA in WA. This suggests the acquisition of the SCCmec element has given this clone a selective advantage. Although the Queensland clone is believed to have been introduced into WA in 2001 [22], PVL positive ST93-MSSA was identified as the most prevalent $S$. aureus clone in WA's remote indigenous communities in surveys performed in the mid 1990s. Although found in an environment of high $\beta$-lactam use a methicillin-resistant variant of ST93-MSSA was not found in WA during these surveys.

WA1, WA2 and WA3 are PVL negative and do not harbor multiple virulence genes (Tables 1 ). Similarly the successful Queensland clone, although PVL positive, carries almost no other exotoxin genes and no additional resistance genes. Although most other WA CA-MRSA clones are also PVL negative, many of these clones have acquired multiple resistance and/or virulence determinants (Tables 1). For example WA78 (ST188-IVa [2B]/ t315) in addition to mecA and blaZ, harbors aacA-aphD, tet $K$ and cat and is phenotypically resistant to erythromycin, trimethoprim and ciprofloxacin; WA64 (ST5-IVa $[2 \mathrm{~B}] / \mathrm{t} 3778)$ has acquired $s e A$ enterotoxin genes and edinA and $l u k F-P V$ lukS-PV virulence genes; and WA62 (ST923[ST8slv]-IVa [2B]/t1635) harbors $s e D+s e J+s e R$ and $s e K+s e Q$ enterotoxin genes and $l u k F-P V l u k S-P V$. The acquisition of multiple resistance and/or virulence genes may have come at a high fitness cost as none of these clones have established a niche in the WA community.

As WA1, WA2 and WA3 CA-MRSA lack PVL as well as other virulence genes that are found in pandemic international CA-MRSA clones, such ACME in USA300, the epidemiology of CA-MRSA disease in WA is different to other regions. Outside of WA the majority of diseases related to CA-MRSA infection are severe skin and soft tissue infections such as soft tissue abscess, carbuncles and furuncles. Many of these infections have occurred in healthy individuals, especially children and adolescents, usually via skin-to-skin contact [41]. In WA the majority of CA-MRSA related diseases were initially associated with the indigenous population and then other groups normally susceptible to $S$. aureus infections such as the elderly. As the original WA CA-MRSA are PVL negative, many of these infections were superficial skin infections such as impetigo. However with the introduction of the PVL-positive Queensland CA-MRSA clone more severe skin and soft tissues infections have been observed.

The limitation of this study is that only the initial isolate of each PFGE pulsotype was included in the study. To determine if the successful CA-MRSA clones found in the WA community are evolving the genetic profiles of subsequent isolates need to be investigated.

\section{Conclusions}

In conclusion although the vertical and horizontal transmission of SCCmec elements into $S$. aureus has 
occurred on multiple occasions in the WA community only three WA CA-MRSA clones have found an ecological niche. These three PVL negative clones harbor few additional resistance and virulence genes which paradoxically may account for their success.

\section{Methods}

\section{Isolates}

The isolates studied are representative of the $83 \mathrm{CA}$ MRSA unique PFGE strains identified in WA from 1989 to 2010 (Figure 3). They include five strains isolated

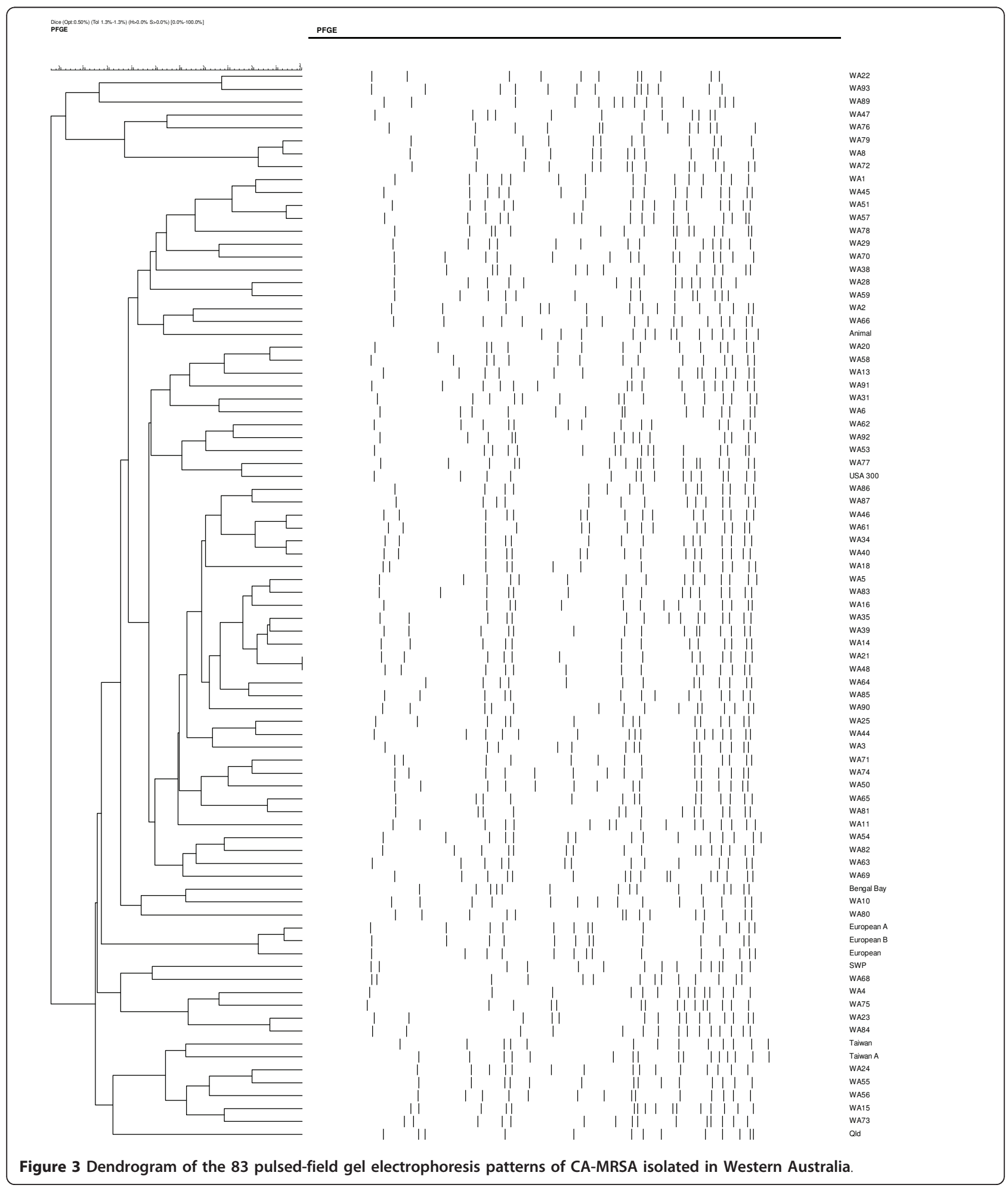


from indigenous inhabitants living in remote WA rural communities in 1989 (WA5 \{WBG7583\} [20]) and 1995 (WA1 \{WBG 8287\}, WA2 \{WB8366\}, WA3 \{WBG8378\}, and WA4 \{WBG8404\} [42]); and 78 strains identified from 24,368 CA-MRSA referred to ACCESS Typing and Research between July 2003 and June 2010.

\section{nuc and mecA}

$S$. aureus species and methicillin resistance was confirmed by the detection of nuc (thermostable extracellular nuclease) and mecA (methicillin resistance) genes by PCR [43].

\section{Susceptibility testing}

An antibiogram was performed by disk diffusion on Mueller-Hinton agar according to the Clinical and Laboratory Standards Institute (CLSI) recommendations [44]. A panel of eight antimicrobial drugs was tested: erythromycin $(15 \mu \mathrm{g})$, tetracycline $(30 \mu \mathrm{g})$, trimethoprim $(5 \mu \mathrm{g})$, ciprofloxacin $(5 \mu \mathrm{g})$, gentamicin $(10 \mu \mathrm{g})$, rifampin $(5 \mu \mathrm{g})$, fusidic acid $(10 \mu \mathrm{g})$, and mupirocin $(5 \mu \mathrm{g})$. CLSI interpretive criteria [45] were used for all drugs except fusidic acid [46] and mupirocin [47].

\section{PVL}

PCR for the detection of PVL determinants was performed as previously described [48].

\section{PFGE}

Electrophoresis of chromosomal DNA was performed as previously described [49], using a contour-clamped homogeneous electric field (CHEF) DR III system (BioRad Laboratories Pty Ltd). Chromosomal patterns were examined visually, scanned with a Quantity One device (Bio-Rad Laboratories Pty Ltd), and digitally analyzed using FPQuest (Bio-Rad Laboratories Pty Ltd). S. aureus strain NCTC 8325 was used as a reference strain.

\section{MLST and spa typing}

Chromosomal DNA for MLST and spa typing was prepared using a DNeasy tissue kit (Qiagen Pty Ltd).

MLST was performed as previously described [50]. The sequences were submitted to http://www.mlst.net/ where an allelic profile was generated and an ST assigned. Clonal complex (CC) was determined using the eBURST V3 algorithm at the same website. Clones that diverged at no more than one of the seven MLST loci were considered to belong to the same CC. Double locus variants (dlvs) were included if the linking single locus variant (slv) was present in the MLST database.

spa typing, a DNA sequenced-based analysis of the protein A gene variable region was performed as previously described [51] using the nomenclature as described on the Ridom website (http://spa.ridom.de/).

\section{SCCmec typing}

The strategy used for $\mathrm{SCCmec}$ typing was as previously described [32]. SCCmec nomenclature is used as proposed by the International Working Group on the Classification of Staphylococcal Cassette Chromosome Elements (IWG-SCC) [52]. Briefly, the structural type is indicated by a Roman numeral, with a lowercase letter indicating the subtype, and the $c c r$ complex and the mec complex are indicated by an Arabic numeral and an uppercase letter respectively in parenthesis. Where there is an extra $c c r$ element, this is indicated by " $\&$ " and an Arabic numeral designating the $c c r$ type. When there is an extra $c c r$ element present whose precise location is unknown it is indicated by an " $\&$ " and $c c r$ number outside the parentheses.

\section{DNA microarray}

Arrays and reagents were obtained from Alere Technologies, Jena Germany. The principle of the assay, related procedures, and a list of targets has been described previously $[53,54]$. An iterated, linear primer elongation was employed for the simultaneous amplification of all targets. An alternative protocol was used for a few isolates in which amplification and labeling was directed by random primers [55]. This method detects target genes for which the binding sites of the primers used in the first protocol were deleted or changed by nucleotide polymorphisms. Target genes included species markers, markers for accessory gene regulator (agr) alleles and capsule types, virulence factors, resistance genes, staphylococcal superantigen-like/exotoxin-like genes (set/ssl genes) and genes encoding adhesion proteins. Probes for mecA, ugp $Q$, $x y l R$, and two probes for mecR were used for SCCmec typing. The last two probes allowed detection and discrimination of untruncated $m e c R$ and $\triangle m e c R$, respectively. Probes for the recombinase genes $c c r A 1, \operatorname{cr} B 1, \operatorname{ccr} A 2, \operatorname{ccr} B 2$, $c c r A 3, c c r B 3, c c r A 4, c c r B 4$, and $c c r C 1$; the fusidc acid resistance marker Q6GD50; and the J region proteins, $d c s$, pls$\mathrm{SCC}$ and the $k d p$-operon were also included.

\section{MRSA Strain Definition}

MRSA strains are defined according to their unique PFGE pulsotype

\section{MRSA Clone Definition}

MRSA clones are defined by the combination of the multilocus sequence type (ST) and the SCCmec type [56]. For instance ST1-SCCmec IVa [2B] is abbreviated as ST1-IVa [2B]. 


\section{Additional material}

\section{Additional file 1: Characterisation of CA-MRSA isolated in Western Australia.}

Additional file 2: DNA Microarray Targets, Primers and Probes.

\section{Acknowledgements}

We gratefully acknowledge the following: the WA Genome Resource Centre, Department of Clinical Immunology and Biochemical Genetics, Royal Perth Hospital for sequencing; the Molecular Biology Laboratory at Royal Perth Hospital for MLST; the Department of Health WA for funding the ACCESS Typing and Research; and the public and private medical microbiology laboratories in Western Australia for referring the isolates.

\section{Author details}

'Australian Collaborating Centre for Enterococcus and Staphylococcus Species (ACCESS) Typing and Research. PathWest Laboratory Medicine - WA, Royal Perth Hospital, Wellington Street, Perth, Western Australia, 6000 Australia. ${ }^{2}$ School of Biomedical Sciences. Curtin University of Technology, GPO Box U1987, Perth, Western Australia, 6000 Australia. ${ }^{3}$ Alere Technologies GmbH, Löbstedter Straße 103-105, D-07749 Jena, Germany.

\section{Authors' contributions}

GC designed the study, analysed and interpreted the data, and drafted the manuscript. SM assisted in the analysis and interpretation of data, and critically revised the manuscript for important intellectual content. JP, HL-T, Y-KC and LE carried out the laboratory procedures. RE critically revised the manuscript for important intellectual content. FGO assisted in the design of the study, analysed and interpreted the data, and critically revised the manuscript for important intellectual content. KJC assisted in the design of the study, analysed and interpreted the data, and critically revised the manuscript for important intellectual content. All authors read and approved the final manuscript.

Received: 29 April 2011 Accepted: 29 September 2011

Published: 29 September 2011

\section{References}

1. Calfee DP, Durbin LJ, Germanson TP, Toney DM, Smith EB, Farr BM: Spread of methicillin-resistant Staphylococcus aureus (MRSA) among household contacts of individuals with nosocomially acquired MRSA. Infect Control Hosp Epidemiol 2003, 24(6):422-426.

2. Salgado CD, Farr BM, Calfee DP: Community-acquired methicillin-resistant Staphylococcus aureus: a meta-analysis of prevalence and risk factors. Clin Infect Dis 2003, 36(2):131-139.

3. Seybold U, Kourbatova EV, Johnson JG, Halvosa SJ, Wang YF, King MD, Ray SM, Blumberg HM: Emergence of community-associated methicillinresistant Staphylococcus aureus USA300 genotype as a major cause of health care-associated blood stream infections. Clin Infect Dis 2006, 42(5):647-656.

4. Patel M, Hoesley CJ, Moser SA, Stamm AM, Baddley JW, Waites KB: Dissemination of community-associated methicillin-resistant Staphylococcus aureus in a tertiary care hospital. South Med J 2008, 101(1):40-45.

5. Okuma K, Iwakawa K, Turnidge JD, Grubb WB, Bell JM, O'Brien FG, Coombs GW, Pearman JW, Tenover FC, Kapi M, et al: Dissemination of new methicillin-resistant Staphylococcus aureus clones in the community. J Clin Microbiol 2002, 40(11):4289-4294.

6. O'Brien FG, Coombs GW, Pearson JC, Christiansen KJ, Grubb WB: Type V staphylococcal cassette chromosome mec in community staphylococci from Australia. Antimicrob Agents Chemother 2005, 49(12):5129-5132.

7. Kobayashi SD, DeLeo FR: An update on community-associated MRSA virulence. Curr Opin Pharmacol 2009, 9(5):545-551.

8. Vandenesch F, Naimi T, Enright MC, Lina G, Nimmo GR, Heffernan $H$, Liassine N, Bes M, Greenland T, Reverdy ME, et al: Community-acquired methicillin-resistant Staphylococcus aureus carrying Panton-Valentine leukocidin genes: worldwide emergence. Emerg Infect Dis 2003, 9(8):978-984
9. Tenover FC, McDougal LK, Goering RV, Killgore G, Projan SJ, Patel JB, Dunman PM: Characterization of a strain of community-associated methicillin-resistant Staphylococcus aureus widely disseminated in the United States. J Clin Microbiol 2006, 44(1):108-118.

10. McDougal LK, Steward CD, Killgore GE, Chaitram JM, McAllister SK, Tenover FC: Pulsed-field gel electrophoresis typing of oxacillin-resistant Staphylococcus aureus isolates from the United States: establishing a national database. J Clin Microbiol 2003, 41(11):5113-5120.

11. Bekkhoucha SN, Cady A, Gautier P, Itim F, Donnio PY: A portrait of Staphylococcus aureus from the other side of the Mediterranean Sea: molecular characteristics of isolates from Western Algeria. Eur J Clin Microbiol Infect Dis 2009, 28(5):553-555.

12. Udo EE, O'Brien FG, Al-Sweih N, Noronha B, Matthew B, Grubb WB: Genetic lineages of community-associated methicillin-resistant Staphylococcus aureus in Kuwait hospitals. J Clin Microbiol 2008, 46(10):3514-3516.

13. Boyle-Vavra S, Ereshefsky B, Wang CC, Daum RS: Successful multiresistant community-associated methicillin-resistant Staphylococcus aureus lineage from Taipei, Taiwan, that carries either the novel Staphylococcal chromosome cassette mec (SCCmec) type VT or SCCmec type IV. J Clin Microbiol 2005, 43(9):4719-4730.

14. Coombs GW, Nimmo GR, Pearson JC, Christiansen KJ, Bell JM, Collignon PJ, McLaws ML: Prevalence of MRSA strains among Staphylococcus aureus isolated from outpatients, 2006. Commun Dis Intell 2009, 33(1):10-20.

15. Collignon P, Gosbell I, Vickery A, Nimmo G, Stylianopoulos T, Gottlieb T: Community-acquired meticillin-resistant Staphylococcus aureus in Australia. Australian Group on Antimicrobial Resistance. Lancet 1998, 352(9122):145-146

16. Riley D, MacCulloch D, Morris AJ: Methicillin-resistant S. aureus in the suburbs. N Z Med J 1998, 111(1060):59.

17. Ellington MJ, Ganner M, Warner M, Cookson BD, Kearns AM: Polyclonal multiply antibiotic-resistant methicillin-resistant Staphylococcus aureus with Panton-Valentine leucocidin in England. J Antimicrob Chemother 2010, 65(1):46-50.

18. Nimmo GR, Coombs GW: Community-associated methicillin-resistant Staphylococcus aureus (MRSA) in Australia. Int J Antimicrob Agents 2008, 31(5):401-410.

19. Tristan A, Bes M, Meugnier $H$, Lina G, Bozdogan B, Courvalin P, Reverdy ME, Enright MC, Vandenesch F, Etienne J: Global distribution of PantonValentine leukocidin-positive methicillin-resistant Staphylococcus aureus, 2006. Emerg Infect Dis 2007, 13(4):594-600.

20. Udo EE, Pearman JW, Grubb WB: Genetic analysis of community isolates of methicillin-resistant Staphylococcus aureus in Western Australia. J Hosp Infect 1993, 25(2):97-108.

21. Coombs GW, Nimmo GR, Bell JM, Huygens F, O'Brien FG, Malkowski MJ, Pearson JC, Stephens AJ, Giffard PM: Genetic diversity among community methicillin-resistant Staphylococcus aureus strains causing outpatient infections in Australia. J Clin Microbio/ 2004, 42(10):4735-4743.

22. Coombs GW, Pearson JC, O'Brien FG, Murray RJ, Grubb WB, Christiansen KJ: Methicillin-resistant Staphylococcus aureus clones, Western Australia. Emerg Infect Dis 2006, 12(2):241-247.

23. Maguire GP, Arthur AD, Boustead PJ, Dwyer B, Currie BJ: Emerging epidemic of community-acquired methicillin-resistant Staphylococcus aureus infection in the Northern Territory. Med J Aust 1996, 164(12):721-723.

24. Vlack S, Cox L, Peleg AY, Canuto C, Stewart C, Conlon A, Stephens A, Giffard P, Huygens F, Mollinger A, et al: Carriage of methicillin-resistant Staphylococcus aureus in a Queensland Indigenous community. Med J Aust 2006, 184(11):556-559.

25. Stevens CL, Ralph A, McLeod JE, McDonald MI: Community-acquired methicillin-resistant Staphylococcus aureus in Central Australia. Commun Dis Intell 2006, 30(4):462-466.

26. Coombs GW, Van Gessel H, Pearson JC, Godsell MR, O'Brien FG, Christiansen $\mathrm{KJ}$ : Controlling a multicenter outbreak involving the New York/Japan methicillin-resistant Staphylococcus aureus clone. Infect Control Hosp Epidemiol 2007, 28(7):845-852.

27. Dailey L, Coombs GW, O'Brien FG, Pearman JW, Christiansen K, Grubb WB, Riley TV: Methicillin-resistant Staphylococcus aureus, Western Australia. Emerg Infect Dis 2005, 11(10):1584-1590.

28. Kennedy AD, Otto M, Braughton KR, Whitney AR, Chen L, Mathema B, Mediavilla JR, Byrne KA, Parkins LD, Tenover FC, et al: Epidemic community-associated methicillin-resistant Staphylococcus aureus: recent 
clonal expansion and diversification. Proc Natl Acad Sci USA 2008, 105(4):1327-1332.

29. O'Brien FG, Lim TT, Chong FN, Coombs GW, Enright MC, Robinson DA, Monk A, Said-Salim B, Kreiswirth BN, Grubb WB: Diversity among community isolates of methicillin-resistant Staphylococcus aureus in Australia. J Clin Microbiol 2004, 42(7):3185-3190.

30. van Wamel WJ, Rooijakkers SH, Ruyken M, van Kessel KP, van Strijp JA: The innate immune modulators staphylococcal complement inhibitor and chemotaxis inhibitory protein of Staphylococcus aureus are located on beta-hemolysin-converting bacteriophages. J Bacteriol 2006 188(4):1310-1315.

31. Monecke S, Ehricht R, Slickers P, Tan HL, Coombs G: The molecular epidemiology and evolution of the Panton-Valentine leukocidin-positive, methicillin-resistant Staphylococcus aureus strain USA300 in Western Australia. Clin Microbiol Infect 2009, 15(8):770-776.

32. Coombs GW, Monecke S, Ehricht R, Slickers P, Pearson JC, Tan HL, Christiansen KJ, O'Brien FG: Differentiation of clonal complex 59 community-associated methicillin-resistant Staphylococcus aureus in Western Australia. Antimicrob Agents Chemother 2010, 54(5):1914-1921.

33. Monecke S, Kanig H, Rudolph W, Muller E, Coombs G, Hotzel H, Slickers P, Ehricht R: Characterisation of Australian MRSA Strains ST75- and ST883MRSA-IV and Analysis of Their Accessory Gene Regulator Locus. PLOS One 2010, 5(11):e14025.

34. van Loo I, Huijsdens X, Tiemersma E, de Neeling A, van de SandeBruinsma N, Beaujean D, Voss A, Kluytmans J: Emergence of methicillinresistant Staphylococcus aureus of animal origin in humans. Emerg Infect Dis 2007, 13(12):1834-1839.

35. Maguire GP, Arthur AD, Boustead PJ, Dwyer B, Currie BJ: Clinical experience and outcomes of community-acquired and nosocomial methicillin-resistant Staphylococcus aureus in a northern Australian hospital. J Hosp Infect 1998, 38(4):273-281.

36. Mak DB, O'Neill LM, Herceg A, MCFarlane H: Prevalence and control of trachoma in Australia, 1997-2004. Commun Dis Intell 2006, 30(2):236-247.

37. O'Brien FG, Coombs GW, Pearman JW, Gracey M, Moss F, Christiansen KJ, Grubb WB: Population dynamics of methicillin-susceptible and -resistant Staphylococcus aureus in remote communities. J Antimicrob Chemother 2009, 64(4):684-693.

38. Nubel U, Roumagnac $\mathrm{P}$, Feldkamp M, Song JH, Ko KS, Huang YC, Coombs G, Ip M, Westh H, Skov R, et al: Frequent emergence and limited geographic dispersal of methicillin-resistant Staphylococcus aureus. Proc Natl Acad Sci USA 2008, 105(37):14130-14135.

39. Huijsdens XW, van Dijke BJ, Spalburg E, van Santen-Verheuvel MG, Heck ME, Pluister GN, Voss A, Wannet WJ, de Neeling AJ: Community-acquired MRSA and pig-farming. Ann Clin Microbiol Antimicrob 2006, 5:26.

40. Munckhof WJ, Schooneveldt J, Coombs GW, Hoare J, Nimmo GR: Emergence of community-acquired methicillin-resistant Staphylococcus aureus (MRSA) infection in Queensland, Australia. Int I Infect Dis 2003, 7(4):259-264

41. Yamamoto T, Nishiyama A, Takano T, Yabe S, Higuchi W, Razvina O, Shi D: Community-acquired methicillin-resistant Staphylococcus aureus: community transmission, pathogenesis, and drug resistance. J Infect Chemother 2010, 16(4):225-254

42. O'Brien FG, Pearman JW, Gracey M, Riley TV, Grubb WB: Community strain of methicillin-resistant Staphylococcus aureus involved in a hospital outbreak. J Clin Microbiol 1999, 37(9):2858-2862.

43. Costa AM, Kay I, Palladino S: Rapid detection of mecA and nuc genes in staphylococci by real-time multiplex polymerase chain reaction. Diagn Microbiol Infect Dis 2005, 51(1):13-17.

44. CLSI: Performance standards for antimicrobial disk susceptibility tests. 7th ed Approved standard M02-A10 CLSI, Wayne, PA.; 2009.

45. CLSI: Performance standards for antimicrobial susceptibility testing. 19th informational supplement M100-S18 CLSI, Wayne, PA; 2009.

46. CA-SFM: Report of the Comité de l'Antibiogramme de la Société Française de Microbiologie. Clin Microbiol Infect 1996, 2:(S48).

47. Finlay JE, Miller LA, Poupard JA: Interpretive criteria for testing susceptibility of staphylococci to mupirocin. Antimicrob Agents Chemother 1997, 41(5):1137-1139

48. Fey PD, Said-Salim B, Rupp ME, Hinrichs SH, Boxrud DJ, Davis CC, Kreiswirth BN, Schlievert PM: Comparative molecular analysis of community- or hospital-acquired methicillin-resistant Staphylococcus aureus. Antimicrob Agents Chemother 2003, 47(1):196-203.
49. O'Brien FG, Udo EE, Grubb WB: Contour-clamped homogeneous electric field electrophoresis of Staphylococcus aureus. Nat Protoc 2006, 1(6):3028-3033.

50. Enright MC, Day NP, Davies CE, Peacock SJ, Spratt BG: Multilocus sequence typing for characterization of methicillin-resistant and methicillinsusceptible clones of Staphylococcus aureus. J Clin Microbiol 2000, 38(3):1008-1015.

51. Harmsen D, Claus H, Witte W, Rothganger J, Turnwald D, Vogel U: Typing of methicillin-resistant Staphylococcus aureus in a university hospital setting by using novel software for spa repeat determination and database management. J Clin Microbiol 2003, 41(12):5442-5448.

52. Elements IWGotCoSCC: Classification of staphylococcal cassette chromosome mec (SCCmec): guidelines for reporting novel SCCmec elements. Antimicrob Agents Chemother 2009, 53(12):4961-4967.

53. Monecke S, Jatzwauk L, Weber S, Slickers P, Ehricht R: DNA microarraybased genotyping of methicillin-resistant Staphylococcus aureus strains from Eastern Saxony. Clin Microbiol Infect 2008, 14(6):534-545.

54. Monecke S, Slickers P, Ehricht R: Assignment of Staphylococcus aureus isolates to clonal complexes based on microarray analysis and pattern recognition. FEMS Immunol Med Microbiol 2008, 53(2):237-251.

55. Monecke S, Slickers P, Hotzel H, Richter-Huhn G, Pohle M, Weber S, Witte W, Ehricht R: Microarray-based characterisation of a PantonValentine leukocidin-positive community-acquired strain of methicillinresistant Staphylococcus aureus. Clin Microbiol Infect 2006, 12(8):718-728.

56. Enright MC, Robinson DA, Randle G, Feil EJ, Grundmann H, Spratt BG: The evolutionary history of methicillin-resistant Staphylococcus aureus (MRSA). Proc Natl Acad Sci USA 2002, 99(11):7687-7692.

doi:10.1186/1471-2180-11-215

Cite this article as: Coombs et al.: Evolution and diversity of community-associated methicillin-resistant Staphylococcus aureus in a geographical region. BMC Microbiology 2011 11:215.

\section{Submit your next manuscript to BioMed Central and take full advantage of:}

- Convenient online submission

- Thorough peer review

- No space constraints or color figure charges

- Immediate publication on acceptance

- Inclusion in PubMed, CAS, Scopus and Google Scholar

- Research which is freely available for redistribution 